\title{
Copper Induces Vasorelaxation and Antagonizes Noradrenaline -Induced Vasoconstriction in Rat Mesenteric Artery
}

\author{
Yu-Chun Wanga,b,c Chao-Wei Hu ${ }^{a, c}$ Ming-Yu Liu ${ }^{a}$ Hong-Chao Jiang ${ }^{a}$ Rong Huo \\ De-Li Donga
}

${ }^{a}$ Department of Pharmacology (the State-Province Key Laboratories of Biomedicine-Pharmaceutics of China, Key Laboratory of Cardiovascular Research, Ministry of Education), Harbin Medical University, Harbin, 'bepartment of Pharmacology, Qiqihar Medical University, Qiqihar, P.R.China; 'These authors contributed equally to this work

\section{Key Words}

Copper $\bullet$ Vasorelaxation $・$ Vasoconstriction $\bullet$ Hypotension

\begin{abstract}
Background/Aims: Copper is an essential trace element for normal cellular function and contributes to critical physiological or pathological processes. The aim of the study was to investigate the effects of copper on vascular tone of rat mesenteric artery and compare the effects of copper on noradrenaline (NA) and high $\mathrm{K}^{+}$induced vasoconstriction. Methods: The rat mesenteric arteries were isolated and the vessel tone was measured by using multi wire myograph system in vitro. Blood pressure of carotid artery in rabbits was measured by using physiological data acquisition and analysis system in vivo. Results: Copper dose-dependently blunted NA-induced vasoconstriction of rat mesenteric artery. Copper-induced vasorelaxation was inhibited when the vessels were pretreated with NG-nitro-L-arginine methyl ester (L-NAME). Copper did not blunt high $\mathrm{K}^{+}$-induced vasoconstriction. Copper preincubation inhibited NAevoked vasoconstriction and the inhibition was not affected by the presence of L-NAME. Copper preincubation showed no effect on high $\mathrm{K}^{+}$-evoked vasoconstriction. Copper chelator diethyldithiocarbamate trihydrate (DTC) antagonized the vasoactivity induced by copper in rat mesenteric artery. In vivo experiments showed that copper injection (iv) significantly decreased blood pressure of rabbits and NA or DTC injection (iv) did not rescue the copperinduced hypotension and animal death. Conclusion: Copper blunted NA but not high $\mathrm{K}^{+}-$ induced vasoconstriction of rat mesenteric artery. The acute effect of copper on NA-induced vasoconstriction was depended on nitric oxide (NO), but the effect of copper pretreatment on NA-induced vasoconstriction was independed on NO, suggesting that copper affected NAinduced vasoconstriction by two distinct mechanisms.
\end{abstract}

Copyright (C) 2013 S. Karger AG, Basel 


\section{Introduction}

Copper is a necessary trace element for normal cellular function. Copper is involved in critical processes including mitochondrial respiration and antioxidant capacity in the body [1]. Cellular copper levels must be tightly controlled because both copper accumulation and deficiency can induce cardiovascular disorders [2-4]. Many lines of evidences showed that high serum copper was associated with increased cardiovascular risks, for example, copper level of ischemic cardiomyopathy patients was higher than that of the healthy volunteers [5]; serum copper concentration was higher in rheumatic heart disease patients than in controls [6]; Serum copper level in subjects with acute decompensation of chronic heart failure was higher than that with chronic stable heart failure and serum copper was as a marker of inflammation in prediction of short term outcome in high risk patients with chronic heart failure [7].

Copper plays a role in controlling nitric oxide synthase (NOS) and guanylyl cyclase (GC) activity in vessels $[8,9]$. In rat extrapulmonary artery, copper induced endothelium- and concentration-dependent relaxation through potentiating the action of NO [10]. However, several studies showed that copper might induce inhibitory effect on vasorelaxation. Chiarugi et al. reported that, in the presence of fetal bovine serum (FBS), copper impaired endothelium-dependent vasorelaxation in aortic rings [11]. Emsley et al. found that copper caused a concentration-dependent inhibition of relaxation to acetylcholine when aortic rings were pre-incubated with homocysteine [12]. On the other hand, copper was reported to antagonize phenylephrine-induced contraction [13], but it is not clear whether this antagonism is associated with the interaction of copper with NO. Based on the above information, the present study was designed to characterize the effects of copper on vasorelaxation and vasoconstriction of rat mesenteric artery.

\section{Materials and Methods}

The adult Sprague-Dawley rats and adult Japan white rabbits were used in the study. Animals were maintained in appropriate cages in uniform hygienic conditions in the room temperature, fed with standard rat or rabbit chow, and had free access to water. The animals were fasted overnight before experiment. All animal procedures and experiments were approved (Approval No. HRB20130312) by the Institutional Animal Care and Use Committee of Harbin Medical University.

Agents

NG-nitro-L-arginine methyl ester (L-NAME) and sodium diethyldithiocarbamate trihydrate (DTC) were purchased from Sigma-Aldrich. Carbamylcholine chloride (Carbachol) was purchased from Wako pure chemical industries, Ltd (Japan). Noradrenaline bitartrate was purchased from Tianjin Jinyao Amino Acid Co., Ltd (China). Copper chloride $\left(\mathrm{CuCl}_{2}\right)$ was purchased from Tianjin Kemiou Chemical Reagent Co., Ltd (China).

\section{Rat mesenteric artery dissection}

Adult male Sprague-Dawley rats were sacrificed after anesthetized by sodium pentobarbitone (40mg/ $\mathrm{kg}$, ip) [14]. The abdomen was cut open to expose the intestine. Pieces of mesentery with connecting fat and blood vessels were rapidly dissected out and quickly transferred to physiological salt solution (PSS) at room temperature. The PSS solution was prepared according to the following composition (mM): $\mathrm{NaCl}, 130 ; \mathrm{KCl}$, 4.7; $\mathrm{MgSO}_{4} \cdot 7 \mathrm{H}_{2} \mathrm{O}, 1.17 ; \mathrm{KH}_{2} \mathrm{PO}_{4}, 1.18 ; \mathrm{NaHCO}_{3}, 14.9 ; \mathrm{CaCl}_{2}$, 1.6; D-glucose, 5.5, and aerated with $95 \% \mathrm{O}_{2}$ and $5 \% \mathrm{CO}_{2}$. The third order side branches of the mesentery artery were separated from venous and fat tissue in a dish containing PSS solution. The vessels were dissected into 2-mm rings and moved to an organ bath filled with $5 \mathrm{ml}$ fresh PSS solution oxygenated with $95 \% \mathrm{O}_{2}$ and $5 \% \mathrm{CO}_{2}\left(\mathrm{pH} 7.4\right.$ at $\left.37^{\circ} \mathrm{C}\right)$. The small arterial rings were threaded carefully with wires through the lumen and the isometric contractions were measured by multi wire myograph system (model 620 DMT, Danish Myo Technology, Denmark). 
Wang et al.: Vasorelaxant Effect of Copper

Measurement of contractile and relaxant responses of mesentery artery

After dissection, arterial rings were suspended in the organ bath filled with $5 \mathrm{~mL}$ PSS, which was bubbled continuously with $95 \% \mathrm{O}_{2}$ and $5 \% \mathrm{CO}_{2}$, and maintained at $37^{\circ} \mathrm{C}$. Normalization procedure was applied to make the vessel rings from a fully relaxed status to a specified transmural pressure status. The target transmural pressure was $100 \mathrm{mmHg}$. The vessel rings were equilibrated for $1 \mathrm{hr}$ to achieve a stable resting tension. To check the vascular responses to different types of stimulus and re-activate the function of the vessels, we performed the wake-up protocol after the vessel segments had been heated, equilibrated and stretched with the appropriate passive tension. The contractile function of vessel rings was confirmed by $60 \mathrm{mM} \mathrm{KCl}$ before drug application. The function of intact endothelium was determined by using $5 \mu \mathrm{M}$ noradrenaline (NA) to induce vasoconstriction and $10 \mu \mathrm{M}$ carbachol to induce vasorelaxation. In the in vitro experiments, the concentrations of $\mathrm{NA}$, carbachol, and $\mathrm{KCl}$ were $5 \mu \mathrm{M}, 10 \mu \mathrm{M}$ and $60 \mathrm{mM}$ respectively.

\section{Measurement of invasive blood pressure in rabbits}

The experiments were carried out according to the procedure described previously [15]. Adult Japan white rabbits (male) weighing 2-2.5 kg were anesthetized by $20 \%$ urethane with an initial dose of $5 \mathrm{~mL} / \mathrm{kg}$ [16]. The neck skin was cut open along the median line and the jugular vein and carotid artery were isolated. Firstly, the jugular vein was cannulated for administration of saline as well as the drug to be tested. After cannulation, the cannulation line was flushed with normal saline to prevent thrombosis. Then, the cephalic end of the carotid artery was tied and the cardiac end was clamped with a bulldog clamp for cannulation. The vessel was cannulated using a cannula pre-filled with heparinized normal saline $(0.5 \mathrm{IU} / \mathrm{mL})$. The other end of the cannula connected to a three-way stopcock/saline filled tuberculin syringe. After cannulation, the bulldog clamp at the cardiac end of the vessel was released slowly, ensuring that there was no bleeding at the cannulation site. The three-way stopcock was connected to the pressure transducer which was connected to the multichannel physiological data acquisition and analysis system (Chengdu Instrument Factory, China) to measure the blood pressure.

The basal blood pressure was recorded for $10 \mathrm{~min}$, then $10 \mathrm{mg} / \mathrm{kg} \mathrm{CuCl}_{2}$ (iv) was injected and the blood pressure was monitored, if no change of blood pressure was observed, the dose of $\mathrm{CuCl}_{2}$ was cumulated to $15 \mathrm{mg} / \mathrm{kg}$. When the blood pressure decreased significantly, NA $(0.1 \mathrm{mg} / \mathrm{kg}$, iv) or $40 \mathrm{mg} / \mathrm{kg}$ DTC (iv) was injected and the blood pressure was further recorded.

Statistical analysis

Data are presented as mean \pm SEM, and significance was determined using Student $t$ test directed by SigmaStat Software. $P<0.05$ was considered significant.

\section{Results}

Copper induced NO-dependent relaxation in NA but not high $\mathrm{K}^{+}$-evoked contraction in rat mesenteric artery in vitro

Previous study showed that copper induced vasorelaxation of rat pulmonary artery [10]. We examined the effects of copper on vasoactivity of rat mesenteric artery in vitro. As shown in Fig. 1A, after validating the intactness of artery by NA and carbachol, different concentrations of copper were applied to the mesenteric artery pre-contracted by NA. Copper dose-dependently induced vasorelaxation of rat mesenteric artery. However, when the arteries were pretreated with the endothelial nitric oxide synthase (eNOS) inhibitor L-NAME, copper-induced vasorelaxation was significantly inhibited. The analyzed data showed that the copper concentration-response curve shifted to the right in the presence of L-NAME (the right panel in Fig. 1A).

High $\mathrm{K}^{+}$could also induce vasoconstriction of rat mesenteric artery, but copper showed no significant effect on $\mathrm{K}^{+}$-induced vasoconstriction (Fig. 1B).

Copper preincubation inhibited NA -evoked contraction in rat mesenteric artery in vitro

After validating the intactness of artery by NA and carbachol treatment, the mesenteric arteries were pretreated with different concentrations of copper, then, were induced to 
Fig. 1. Copper induced NO-dependent relaxation in NA but not $\mathrm{KCl}$-evoked contraction in rat mesenteric artery in vitro. (A) Copper induced NO-dependent relaxation in NA-evoked contraction in rat mesenteric artery. NA, Noradrenaline; Carb, Carbachol. $5 \mu \mathrm{M}$ NA and $10 \mu \mathrm{M}$ Carb were used. (B) Copper showed no significant effect on high $\mathrm{K}^{+}(60 \mathrm{mM})$-induced vasoconstriction. $\mathrm{n}=6$ in each group.

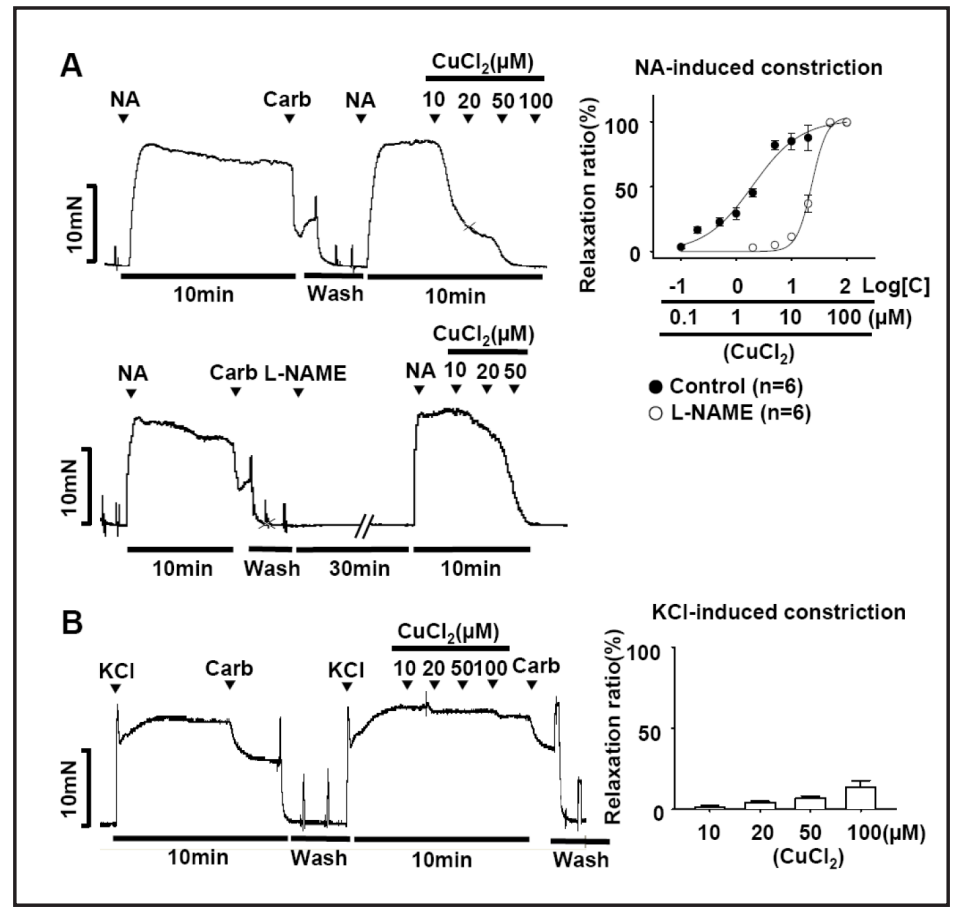

Fig. 2. Copper preincubation inhibited NA -evoked contraction in rat mesenteric artery in vitro. In $\mathrm{A}(\mathrm{c})$ panel, the inhibition of NAinduced vasoconstriction by copper $(50 \mathrm{uM})$ pretreatment manifested transient contraction in some artery samples, or complete loss of contraction in other artery samples. The representative recording of the complete loss of contraction was shown in the enlarged frame. NA, Noradrenaline; Carb, Carbachol. $5 \mu \mathrm{M}$ NA and $10 \mu \mathrm{M}$ Carb were used. $\mathrm{n}=6$.

A (a)

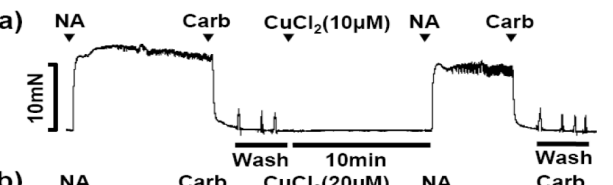

(b)

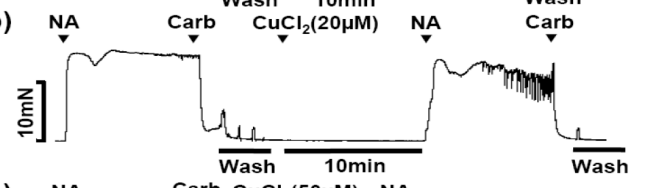

(c)
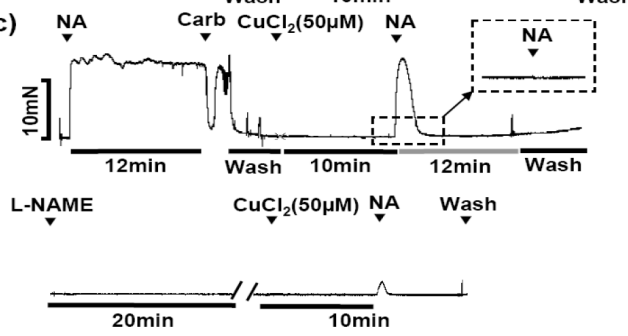

(d)

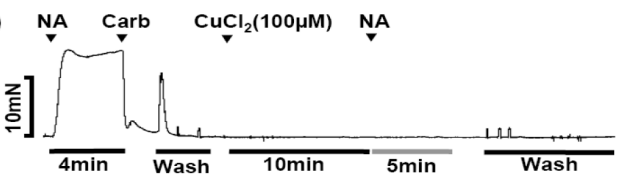

B

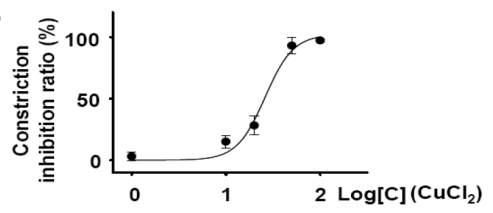

contract by NA treatment. With the increasing of copper concentration, the NA-induced vasoconstriction was gradually inhibited (Fig. 2A). As shown in Fig. 2A(c), the inhibition of NA-induced vasoconstriction by copper $(50 \mathrm{uM})$ pre-treatment manifested transient contraction in some artery samples, or complete loss of contraction in other artery samples. 
Fig. 3. Copper preincubation showed no effect on high $\mathrm{K}^{+}(60 \mathrm{mM})$-evoked contraction in rat mesenteric artery in vitro. Carb, Carbachol. $10 \mu \mathrm{M}$ Carb was used. $\mathrm{n}=6$ in each group.

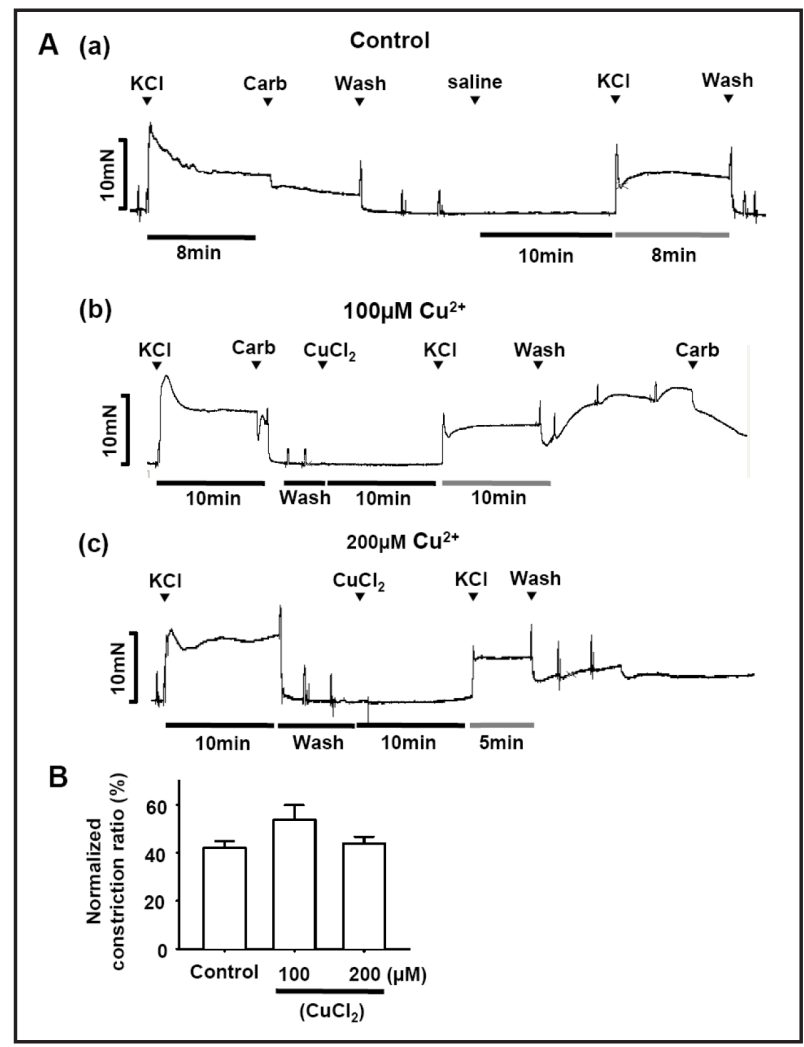

In the presence of L-NAME, copper (50 uM) pretreatment still inhibited NA-induced vasoconstriction, indicating that the antagonism of NA-induced vasoconstriction by copper was not related to nitric oxide (NO). The dose-response curve of inhibition of NA-induced vasoconstriction by copper pretreatment was shown in Fig. 2B.

Copper preincubation showed no effect on high $\mathrm{K}^{+}$-evoked contraction in rat mesenteric artery in vitro

High $\mathrm{K}^{+}$induces vasoconstriction through depolarizing membrane potential of smooth muscle cells. Compared with the control group, copper at concentrations as high as 100, 200 $\mu \mathrm{M}$ showed no significant inhibition on high $\mathrm{K}^{+}$induced-vasoconstriction in rat mesenteric artery (Fig. 3).

Copper chelator DTC antagonized the vasoactivity of copper in rat mesenteric artery in vitro

Diethyldithiocarbamate (DTC) is a potent copper-chelating agent. We used DTC to study the mesenteric artery vasoactivity when copper was antagonized. DTC at $50 \mu \mathrm{M}$ concentrations did not affect the vessel basal tension (Fig. 4A(a)). DTC (50 $\mu \mathrm{M})$ induced further vasoconstriction after the vessel was constricted by NA treatment (Fig. 4A(b)). In the presence of DTC $(50 \mu \mathrm{M})$, copper-induced vasorelaxation was significantly inhibited, the representative recording and analyzed data were shown in Fig. 4B. Copper pretreatment inhibited NA-induced vasoconstriction (Fig. 2), but DTC presence $(50 \mu \mathrm{M})$ reversed the copper-induced inhibition of NA-evoked vasoconstriction. After washout of DTC, copper pretreatment still inhibited NA-induced vasoconstriction. The representative recording and analyzed data were shown in Fig. 4C.

Copper treatment decreased rabbit blood pressure in vivo

The above data showed that copper induced vasorelaxation and antagonized NAinduced vasoconstriction in rat mesenteric artery. Therefore, we further studied the effect 
Fig. 4. Copper chelator diethyldithiocarbamate (DTC) antagonized the vasoactivity of copper in rat mesenteric artery in vitro. (A) DTC at $50 \mu \mathrm{M}$ concentrations did not affect the vessel basal tension (a) but induced further vasoconstriction after the vessel was constricted by NA treatment (b). ${ }^{* *} P<0.01$, vs control. $\mathrm{n}=6$. (B) DTC $(50 \mu \mathrm{M})$ treatment inhibited copperinduced vasorelaxation. ${ }^{*} P<0.05$, ${ }^{* *} P<0.01$ vs copper group. (C) DTC $(50 \mu \mathrm{M})$ treatment reversed the copper-induced inhibition of NA-evoked vasoconstriction. ${ }^{* *} P<0.01$ vs copper group.

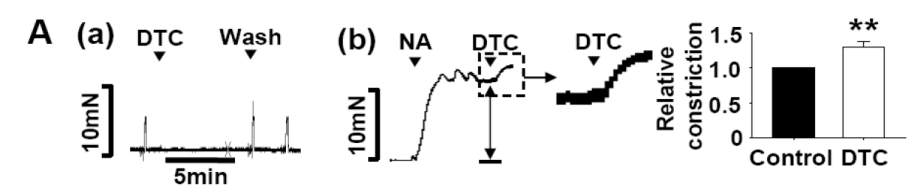

B
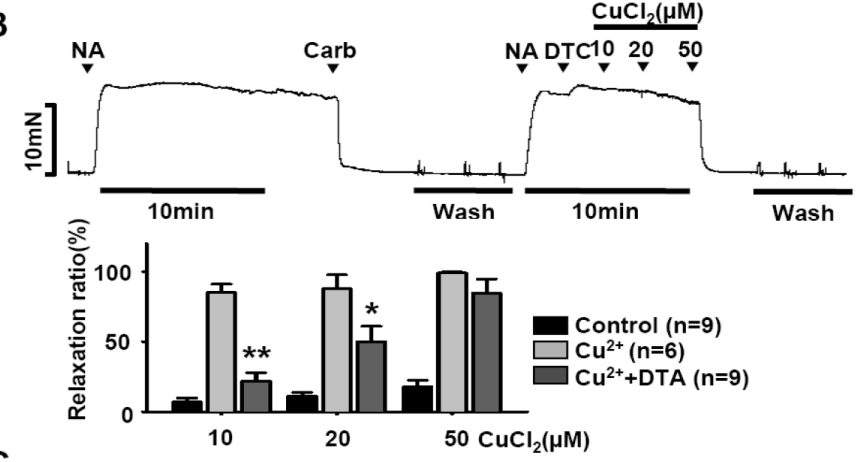

C

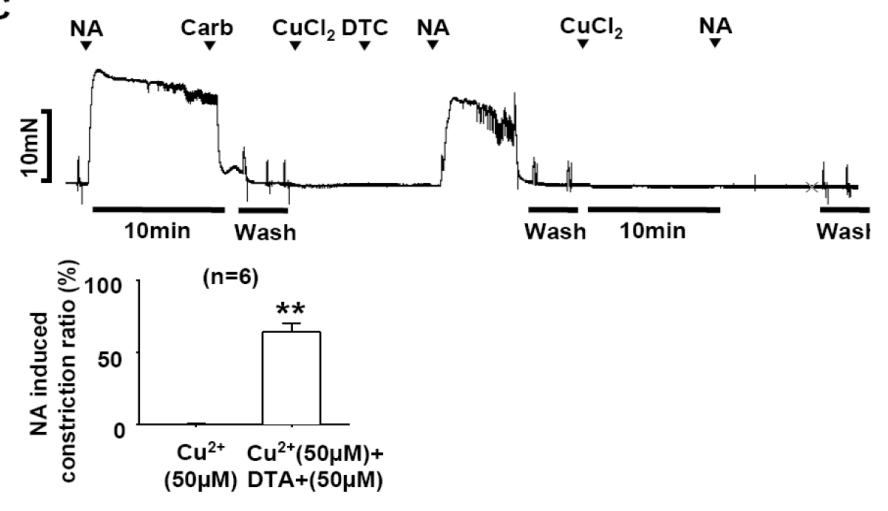

Fig. 5. The representative recording of effect of copper injection on rabbit blood pressure in vivo. $\mathrm{CuCl}_{2}(15 \mathrm{mg} / \mathrm{kg})$ injection (iv) decreased blood pressure. NA $(0.1 \mathrm{mg} / \mathrm{kg})$ injection (iv) did not reverse the copper-induced hypotension and animal death.

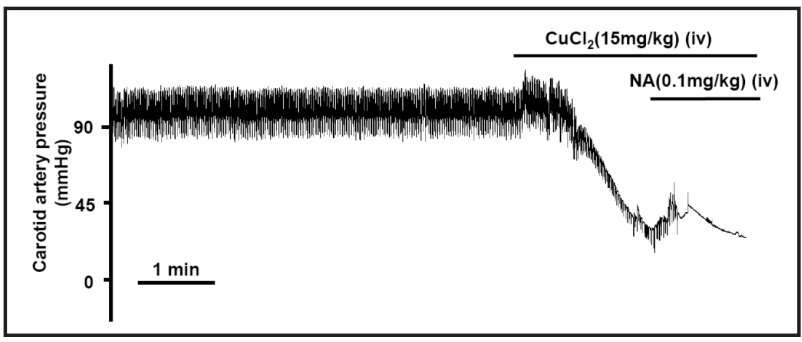

of copper on the blood pressure and whether NA or DTC could rescue the copper-induced hypotension in rabbits in vivo. The basal blood pressure was not altered during $5 \mathrm{~min}$ after $10 \mathrm{mg} / \mathrm{kg} \mathrm{CuCl}{ }_{2}$ injection (iv) in 3 rabbits. When the dose of $\mathrm{CuCl}_{2}$ was increased to $15 \mathrm{mg} / \mathrm{kg}$, significant decrease of blood pressure and subsequent animal death occurred in observed three rabbits, similar to the previous study [17]. NA $(0.1 \mathrm{mg} / \mathrm{kg}$, iv) which increases blood pressure and $40 \mathrm{mg} / \mathrm{kg}$ DTC (iv) which chelates copper did not reverse the copper-induced hypotension and animal death $(n=3$, respectively). The representative recording was shown in Fig. 5.

\section{Discussion}

In the present study, we found that copper relaxed NA but not high $\mathrm{K}^{+}$-induced vasoconstriction of rat mesenteric artery. Copper relaxed NA-induced vasoconstriction dependently of nitric oxide (NO), but pretreatment of copper antagonized NA-induced 
vasoconstriction independently of NO, suggesting that the vasorelaxant effect and the antagonizing vasoconstriction effect of copper were through different mechanisms.

Pretreatment of eNOS inhibitor L-NAME inhibited copper-induced vasorelaxation (Fig. $1 \mathrm{~A})$, which was consistent with the previous reports $[8,10]$. However, in the presence of L-NAME, copper pretreatment still antagonized NA-induced vasoconstriction. We had expected that antagonism of vasoconstriction by copper might be due to its potentiating the action of NO, because the vessel tension is commonly controlled by the balance of vasoconstriction and vasorelaxation. The present results indicated that the antagonism of vasoconstriction by copper was through other mechanisms rather than NO pathway. Ciolek et al. recently reported that copper decreased epinephrine affinity to alpha-adrenoceptor [18]. Since alpha-adrenoceptors in vascular smooth muscle cells are critical for inducing vasoconstriction, we speculated that antagonism of vasoconstriction by copper might be due to its desensitizing alpha-adrenoceptors in the smooth muscle cells.

High $\mathrm{K}^{+}$induces membrane depolarization of smooth muscle cells and subsequent increase of intracellular $\mathrm{Ca}^{2+}$ concentration through activating voltage-dependent $\mathrm{Ca}^{2+}$ channels, thus evokes vasoconstriction. We found that copper showed no effect on $\mathrm{K}^{+}-$ induced vasoconstriction. Although copper had been reported to block different types of voltage-dependent $\mathrm{Ca}^{2+}$ channels in different cells $[19,20]$, the present data showed that copper did not inhibit voltage-dependent $\mathrm{Ca}^{2+}$ channels responsible for regulating vessel tone in rat mesenteric artery.

Copper exists in the blood in two forms, copper in free and copper in binding to ceruloplasmin or other proteins such as transcuperin, albumin, and amino acids. The excess copper, particularly the free fraction, may lead to tissue injury due to pro-oxidant or other effects. A clinically significant change in the free copper concentration may not be detected through measuring total copper concentrations. Serum copper concentration has been reported to increase in variety of cardiovascular diseases [5-7]. But these works measured the total copper but not the free copper concentrations, thus, the real status of free copper in the serum was still undetermined in the cardiovascular diseases with increased serum copper. In the present study, copper at $10 \mu \mathrm{M}$ concentrations had shown significant effect on the vasoactivity. We were not sure whether this concentration was relevant to the free copper level in the diseased states, but it should be relevant to copper intoxication. Our in vivo experiment showed that acute copper administration induced severe hypotension, which was consistent to the in vitro data that copper induced vasorelaxation and antagonized NA-induced vasoconstriction. The results that NA injection did not rescue copper-induced hypotension were also in agreement with the data that copper desensitized contractile response to NA in mesenteric artery in vitro.

DTC is a potent chelator of copper. Broner et al. found that DTC injection increased the blood pressure of rabbits with induced Escherichia coli sepsis [21]. They thought the mechanism was that DTC blocked endothelium-derived relaxing factors (EDRF) including NO. Indeed, many studies reported that NO was generated markedly in sepsis animal models and septic patients [22-24]. Based on that copper stimulated NO production [8-10], it was reasonable to speculate that copper might be involved in the vascular hyporeactivity in sepsis through enhancing NO action. However, DTC injection (iv) could not reverse the copperinduced hypotension and could not prevent vascular hyporeactivity to norepinephrine in copper-treated rabbits. Because current techniques can not accurately measure the free rather than total copper concentrations in serum, we did not further evaluate the role of copper in sepsis, which was a limitation of the present study.

\section{Acknowledgements}

This work was supported by the National Basic Research Program of China (grant number 2012CB517803) and the Chang Jiang Scholar Candidates Program for Provincial Universities in Heilongjiang (grant number 2011CJHB002). 
Wang et al.: Vasorelaxant Effect of Copper

\section{References}

1 Rines AK, Ardehali H: Transition metals and mitochondrial metabolism in the heart. J Mol Cell Cardiol 2013;55:50-57.

2 Li Y, Wang L, Schuschke DA, Zhou Z, Saari JT, Kang YJ: Marginal dietary copper restriction induces cardiomyopathy in rats. J Nutr 2005;135:2130-2136.

- 3 Lu J, Pontré B, Pickup S, Choong SY, Li M, Xu H, Gamble GD, Phillips AR, Cowan BR, Young AA, Cooper GJ: Treatment with a copper-selective chelator causes substantive improvement in cardiac function of diabetic rats with left-ventricular impairment. Cardiovasc Diabetol 2013;12:28.

4 Wei H, Zhang WJ, McMillen TS, Leboeuf RC, Frei B: Copper chelation by tetrathiomolybdate inhibits vascular inflammation and atherosclerotic lesion development in apolipoprotein E-deficient mice. Atherosclerosis 2012;223:306-313.

-5 Shokrzadeh M, Ghaemian A, Salehifar E, Aliakbari S, Saravi SS, Ebrahimi P: Serum zinc and copper levels in ischemic cardiomyopathy. Biol Trace Elem Res 2009;127:116-123.

6 Kosar F, Sahin I, Acikgöz N, Aksoy Y, Kucukbay Z, Cehreli S: Significance of serum trace element status in patients with rheumatic heart disease: a prospective study. Biol Trace Elem Res 2005;107:1-10. Malek F, Jiresova E, Dohnalova A, Koprivova H, Spacek R: Serum copper as a marker of inflammation in prediction of short term outcome in high risk patients with chronic heart failure. Int J Cardiol 2006;113:e51-53.

8 Plane F, Wigmore S, Angelini GD, Jeremy JY: Effect of copper on nitric oxide synthase and guanylyl cyclase activity in the rat isolated aorta. Br J Pharmacol 1997;121:345-350.

-9 Demura Y, Ameshima S, Ishizaki T, Okamura S, Miyamori I, Matsukawa S: The activation of eNOS by copper ion $\left(\mathrm{Cu}^{2+}\right)$ in human pulmonary arterial endothelial cells (HPAEC). Free Radic Biol Med 1998;25:314-320.

10 Ohnishi T, Ishizaki T, Sasaki F, Ameshima S, Nakai T, Miyabo S, Matsukawa S: The effect of $\mathrm{Cu}^{2+}$ on rat pulmonary arterial rings. Eur J Pharmacol 1997;319:49-55.

11 Chiarugi A, Pitari GM, Costa R, Ferrante M, Villari L, Amico-Roxas M, Godfraind T, Bianchi A, Salomone S: Effect of prolonged incubation with copper on endothelium-dependent relaxation in rat isolated aorta. Br J Pharmacol 2002;136:1185-1193.

12 Emsley AM, Jeremy JY, Gomes GN, Angelini GD, Plane F: Investigation of the inhibitory effects of homocysteine and copper on nitric oxide-mediated relaxation of rat isolated aorta. Br J Pharmacol 1999;126:1034-1040.

13 Yan M, Liu DL, Chua YL, Chen C, Lim YL : Effects of micromolar concentrations of manganese, copper, and zinc on alpha1-adrenoceptor-mediating contraction in rat aorta. Biol Trace Elem Res 2001;82:159-166.

14 De-Matos IM, Talvani A, Rocha 00, Freire-Maia L, Teixeira MM: Evidence for a role of mast cells in the lung edema induced by Tityus serrulatus venom in rats. Toxicon 2001;39:863-867.

15 Parasuraman S, Raveendran R: Measurement of invasive blood pressure in rats. J Pharmacol Pharmacother 2012;3:172-177.

-16 Fang B, Li XM, Sun XJ, Bao NR, Ren XY, Lv HW, Ma H: Ischemic Preconditioning Protects against Spinal Cord Ischemia-Reperfusion Injury in Rabbits by Attenuating Blood Spinal Cord Barrier Disruption. Int J Mol Sci 2013;14:10343-10354.

17 Rhee HM, Dunlap M: Acute cardiovascular toxic effects of copper in anesthetized rabbits. Neurotoxicology 1990;11:355-360.

18 Ciolek J, Maïga A, Marcon E, Servent D, Gilles N: Pharmacological characterization of zinc and copper interaction with the human alpha(1A)-adrenoceptor. Eur J Pharmacol 2011;655:1-8.

19 Lu L, Wang C, Gao X, Xu P, Wang J, Wang Q, Cheng J, Xiao H: Effects of copper on T-type Ca ${ }^{2+}$ channels in mouse spermatogenic cells. J Membr Biol 2009;227:87-94.

20 Castelli L, Tanzi F, Taglietti V, Magistretti J: $\mathrm{Cu}^{2+}, \mathrm{Co}^{2+}$, and $\mathrm{Mn}^{2+}$ modify the gating kinetics of high-voltageactivated Ca2+ channels in rat palaeocortical neurons. J Membr Biol 2003;195:121-136.

-21 Broner CW, Shenep JL, Stokes DC, Fairclough D, Hildner WK, Storgion SA, Rehg JE: Reversal of dopaminerefractory septic shock by diethyldithiocarbamate, an inhibitor of endothelium-derived relaxing factor. J Infect Dis 1993;167:141-147.

-22 Santos SS, Brunialti MK, Rigato O, Machado FR, Silva E, Salomao R: Generation of nitric oxide and reactive oxygen species by neutrophils and monocytes from septic patients and association with outcomes. Shock 2012;38:18-23.

23 Wang L, Taneja R, Razavi HM, Law C, Gillis C, Mehta S: Specific role of neutrophil inducible nitric oxide synthase in murine sepsis-induced lung injury in vivo. Shock 2012;37:539-547.

24 Kandasamy K, Prawez S, Choudhury S, More AS, Ahanger AA, Singh TU, Parida S, Mishra SK: Atorvastatin prevents vascular hyporeactivity to norepinephrine in sepsis: role of nitric oxide and $\alpha_{1}$-adrenoceptor mRNA expression. Shock 2011;36:76-82. 